\title{
Triboelectric Nanogenerator Powered Electrowetting-on- Dielectric Actuator for Concealed Aquatic Microbots
}

\author{
Dongyue Jiang ${ }^{a}{ }^{*}$, Zeng Fan ${ }^{b}$, Hongchen Wang ${ }^{a}$, Minyi Xuc, + , Guijun Chen ${ }^{a}$, Yongchen Song ${ }^{a}$, and \\ Zhong Lin Wangd,e, \\ ${ }^{a}$ Key Laboratory of Ocean Energy Utilization and Energy Conservation of Ministry of Education, \\ Dalian University of Technology, China 116024 \\ b School of Physics, Dalian University of Technology, China 116024 \\ c Marine Engineering College, Dalian Maritime University, Liaoning Province, China 116026 \\ d Beijing Institute of Nanoenergy and Nanosystems, Chinese Academy of Sciences, Beijing, China \\ 100083 \\ e School of Materials Science and Engineering, Georgia Institute of Technology, Atlanta, GA 30332, \\ USA
}

\section{Supporting Information}

\footnotetext{
* Correspondence and requests for materials should be addressed to D.Y.J. (email: jiangdy@dlut.edu.cn), M.Y.X. (email: xuminyi@dlmu.edu.cn) or Z.L.W. (email: zhong.wang@mse.gatech.edu)
} 


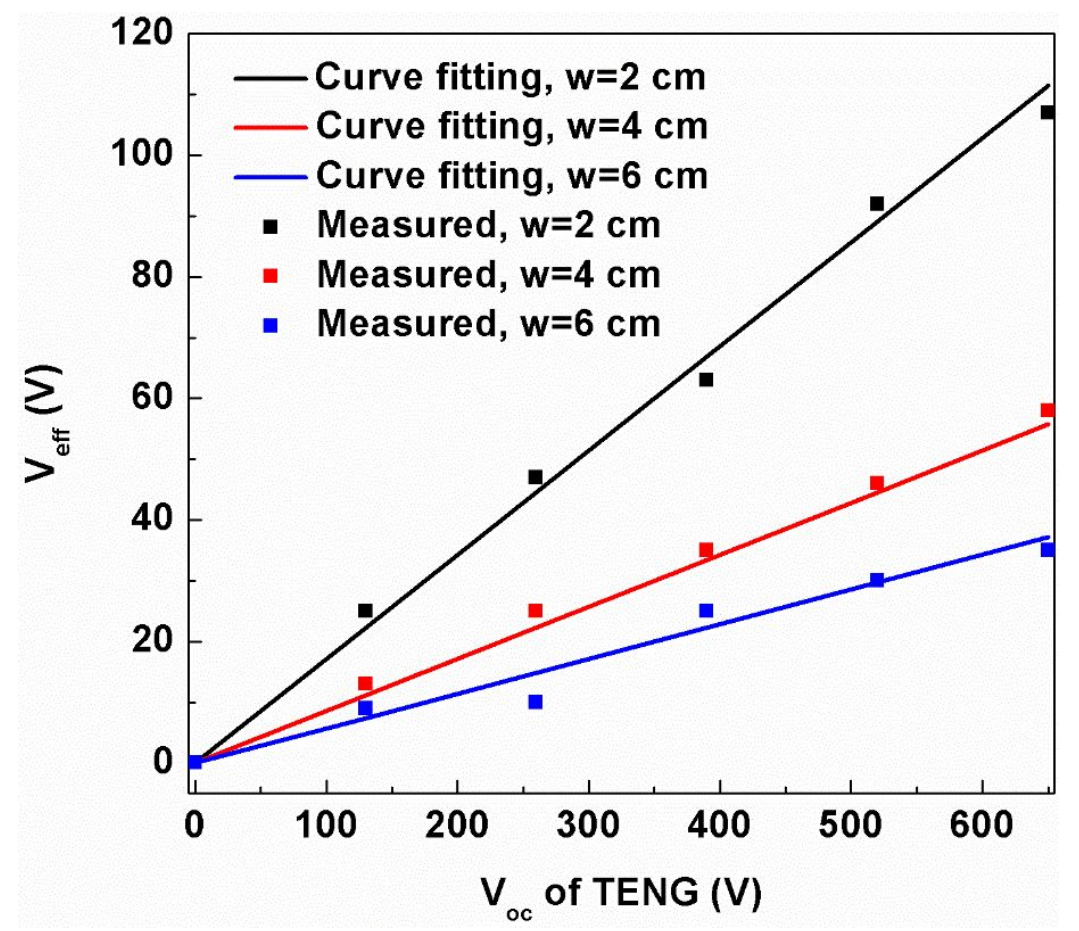

Figure S1. Relationship between $V_{\text {oc }}$ and $V_{\text {eff }}$ at varied actuator electrode width $w$. When TENG is operating, the $\mathrm{V}_{\text {oc }}$ could be read out by electrometer directly. When connecting this $V_{\text {oc }}$ signal to the EWOD actuator with electrode width of $w$, the contact angle can be measured by high speed camera. Based on the information of interfacial tension, relative permittivity and thickness of Teflon, an effective voltage could be predicted from Young-Lippmann's equation. This Veff is found much smaller than Voc, so the authors added a coefficient $p$ to correlate $V_{\text {oc }}$ and $V_{\text {eff }}$ as: $V_{\text {eff }}=V_{o c} /(p w)$. Where $p$ is calculated as a value of 291 and with a unit of $1 / \mathrm{m}$.

\section{Relationship between average wave amplitude and modified contact angle:}

(i) When $\theta_{\min }>90^{\circ}$

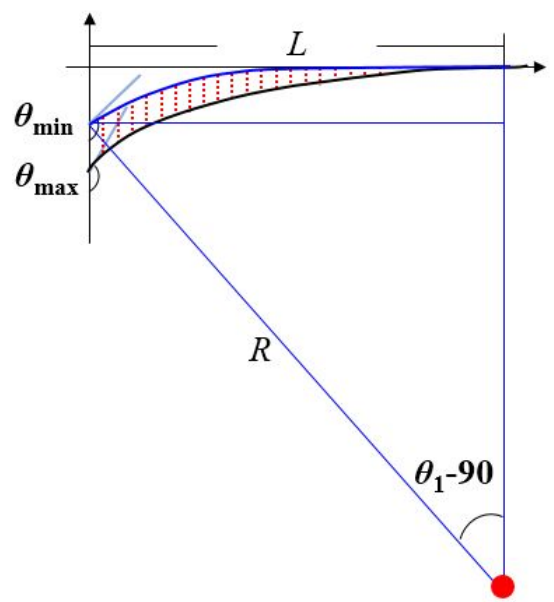

Figure S2. Average wave amplitude estimation when $\theta_{\min }<90^{\circ}$. 
The average amplitude could be obtained by integrating the infinitesimal wave amplitude in the range of $\mathrm{O}-\mathrm{L}$.

$$
\bar{a}=\frac{\int_{0}^{L} a_{i} d L}{L}
$$

From mean value theorem for integrals, $\bar{a}$ can be obtained by dividing the shadow area with $\mathrm{L}$. The shadow area can be attained by the following equation.

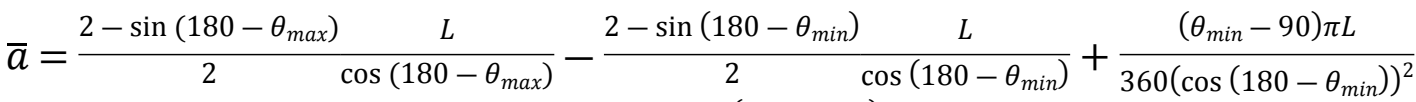

$$
\begin{aligned}
& -\frac{\left(\theta_{\max }-90\right) \pi L}{360\left(\cos \left(180-\theta_{\max }\right)\right)^{2}}
\end{aligned}
$$

Where $\vartheta_{\max }$ is initial contact angle, $110^{\circ}$.

(ii) When $\theta_{\min }=90^{\circ}$, the contact line reaches straight as shown in the figure below and the average wave amplitude can be estimated from:

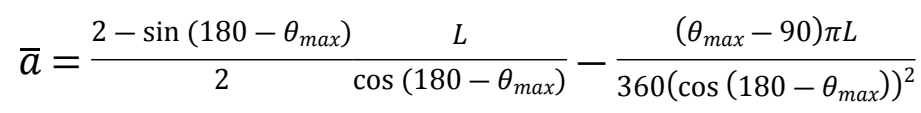

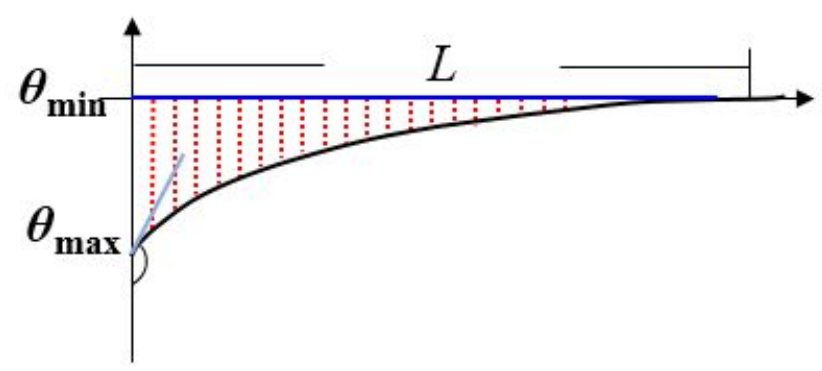

Figure S3. Average wave amplitude estimation when $\theta_{\min }=90^{\circ}$.

(iii) When $\theta_{\min }<90^{\circ}$, the average wave amplitude can be calculated by:

$$
\begin{gathered}
\bar{a}=\frac{2-\sin \left(180-\theta_{\max }\right) \quad L}{2 \cos \left(180-\theta_{\max }\right)}-\frac{\left(\theta_{\max }-90\right) \pi L}{360\left(\cos \left(180-\theta_{\max }\right)\right)^{2}}+\frac{2-\sin \left(\theta_{\min }\right)}{2} \quad L \\
\frac{\left(90-\theta_{\min }\right) \pi L}{360\left(\cos \left(\theta_{\min }\right)\right)^{2}}
\end{gathered}
$$




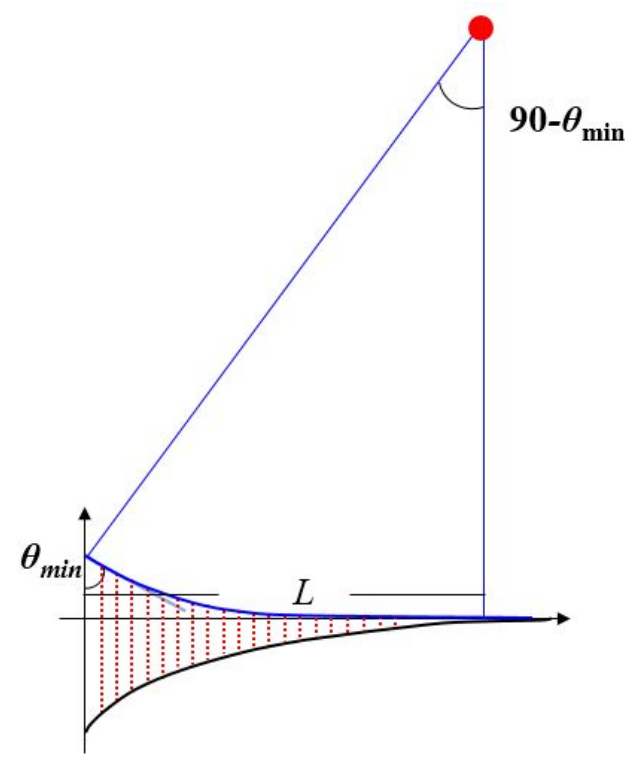

Figure S4. Average wave amplitude estimation when $\theta_{\min }<90^{\circ}$.

\section{Measurement of actuation force:}

The actuation force measurement is based on the setup shown below:

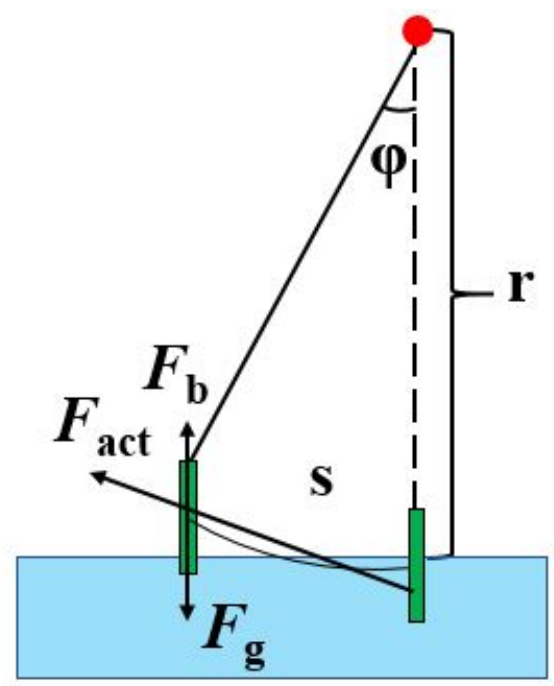

Figure S5. Experimental setup for actuation force measurement.

In this setup, the EWA is connected with a thin nickel wire $(0.025 \mathrm{~mm}$ diameter $)$ and the mass of the wire can be neglected. The top end of the nickel wire is mounted to a rod. The distance between the fixed point and the propeller centroid is $r$. When the capillary wave is generated by TENG, the actuator deflects for a certain distance $s$ and the angle between original and current position is $\varphi$. 
When the actuator stabilizes, the force balance can be given by moment equilibrium and the $F_{\text {act }}$ can be calculated by:

$$
F_{\text {act }}=(m g-\rho g V) \sin (\varphi)
$$

Where the deflection angle $\varphi$ can be obtained by Eq. s2:

$$
\sin (\varphi)=\frac{s}{\sqrt{s^{2}+r^{2}}}
$$

\section{Frequency dependent average wave amplitude:}

For improving the accuracy of the presented model, the frequency dependent average wave amplitude is firstly measured, followed by a curve fitting as shown in Figure S6.

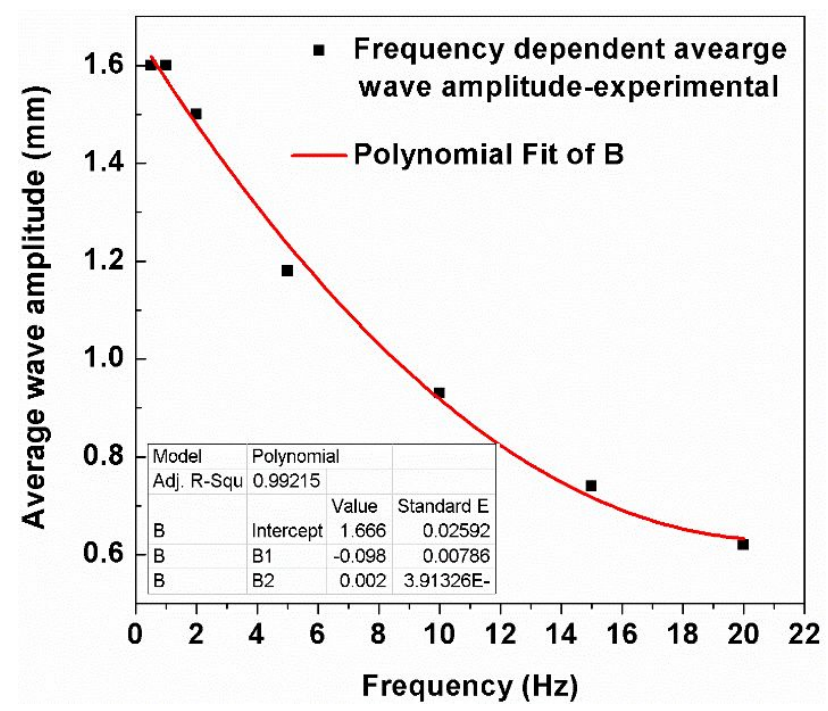

Figure S6. Frequency dependent average wave amplitude. The measured relationship is curve fitted by a polynomial function and the coefficients of the function is shown in the inset of Figure $\mathrm{S} 6$. 